\title{
"Analysis" In Teaching With Cases: A Revisit To Bloom's Taxonomy Of Learning Objectives
}

Nabil Y. Razzouk, (Email: nabil@razzouks.com), California State University Jay N. Razzouk, Pepperdine University

\begin{abstract}
"Analysis", the core of graduate business education is probably one of the least understood and most misrepresented learning activities. Everyone seems to claim that they know it, yet most seem unable to actually define it or practice it. This paper reviews Bloom's taxonomy of learning objectives with a special focus on "analysis." More specifically, the paper seeks to present a more profound perspective of "analysis" and "synthesis" as taught and learned in a typical business course that utilizes case studies. The authors take more of an epidemiological perspective to case analysis which goes beyond the mere rehash of case facts to developing an understanding of all the communication elements in the case and the relationship between the elements for the purpose of synthesizing a new picture that was not obvious before. The paper presents a useful framework for analyzing business cases and recommending and implementing viable strategic options.
\end{abstract}

\section{INTRODUCTION}

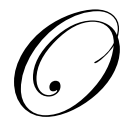

ne of the most common words in business is "Analysis." It is no surprise then to see the word creep into every area of business education. Courses, textbooks, chapters, journal titles, and published literature present analysis as an important staple of every area of business decision making. Marketing, Management, and other business strategy courses utilize case studies to simulate actual business experiences and give students an opportunity to apply knowledge and practice decision making. In real life, as it should be in the classroom, analysis is a crucial component of effective decision making. It could be said that analysis is the core of business education. Whether it is in Marketing, Finance, Personnel, general Management, Information Technology, or any other area, students are trained to first of all analyze the situation, understand the issues, identify the alternatives and then select and implement the best options. The effectiveness of the whole process depends on the person's ability to effectively analyze the situation, though we should hasten to note that effective analysis does not in and of itself guarantee good decisions.

Despite its noted significance, analysis itself receives very little attention from modern academia. A general survey of business textbooks of various disciplines (Marketing, Management, Finance, and Information Management) failed to produce a single appropriate definition of "analysis." Where the term "analysis" is presented or discussed, it is typically described rather than defined in such terms as "assessing," "identifying", "examining", or "evaluating." Overall, it seems that either the authors of the textbooks assume students are already familiar with the concept of analysis or the authors do not feel elaborating on analysis is worth much ink. So what is analysis? And how best could analysis be taught or learned in business education?

\section{THE NATURE OF ANALYSIS}

One of the most useful discourses on analysis remains that of Benjamin Bloom, who in 1956 presented it in the context of the hierarchy of learning objectives. Concerned about the changes produced in individuals as a result 
of educational experiences, Bloom (1956) developed a taxonomy comprised of six major levels of learning: knowledge, comprehension, application, analysis, synthesis, and evaluation.

Knowledge, as defined by Bloom involves the recall of specifics and universals, facts and observations, patterns, structure, or setting. It is the most basic level of learning that involves primarily the psychological processes of remembering and does not require further intellectual abilities or skills."

Comprehension represents the lowest level of understanding. It connotes the learner's ability to make use of the material being communicated without necessarily relating it to other material or even understanding its full implications. At its best, comprehension requires the skill of interpretation which involves recognizing the essentials and differentiating them from the less essential portions or from the relatively irrelevant aspects of the communications. This process requires some capability for abstracting generalizations from a set of particulars as well as for weighing and assessing the relative emphasis to be given the different elements in the communication. In these respects, interpretation becomes synonymous with analysis and has characteristics in common with evaluation. One can argue that any significant learning through case studies requires the student to develop a much higher level of comprehension than the mere understanding of words and their meanings. The student must be able to separate between what is relevant and what is not in the context of a particular decision-making situation.

The third class in the hierarchy is Application - the use of abstractions in particular and concrete situations without having to be prompted as to which abstraction is correct or without having to be shown how to use it in that situation. In Comprehension the emphasis is on the grasp of the meaning and intent of the material. In Application it is on remembering and bringing to bear upon given material the appropriate generalizations or principles. It could be argued that the underlying learning premise from case studies is the application of learned cognitions and relationships to specific decision-making situations. Thus, the company and the decision situation become the theatre in which strategies, plans, and tactics are tried or tested. The fact that one may never be able to find exactly how those proposed strategies, plans, and tactics will fare in that situation, this remains the major caveat of case study learning. On the other hand, this pseudo-applied perspective remains one of the next-best approaches to learning by trial and error in a true decision making role.

Analysis, the focus of this paper, emphasizes the breakdown of the communicated material into its constituent parts and detection of the relationships of the parts and of the way they are organized." (Bloom, 1956. P. 144.) To achieve its intended objective in the learning process, Analysis must help the learner achieve the following three tasks:

1. Break the whole communication down into its constituent elements.

2. Examine relationships of the elements to each other or elements to the main idea (thesis). The purpose of this step is to determine the connections and interactions between the various parts.

3. Analyze the structure and organization (e.g., asking what is the form, pattern, or structure used?) and identify the purpose of the message. Recognize the organizational principles, arrangements and structure which hold the communication together.

The last two classes of the taxonomy are Synthesis and Evaluation. Synthesis pertains to bringing together all the elements and parts of the case study material to form a new whole. This process involves arranging or rearranging of the pieces into a new mosaic that was not there before. It requires well developed written and oral communication skills as well as organization capabilities.

At the top of the hierarchy is Evaluation. This process involves the learner making judgments about the value of the information in the case, or processes and methods cited in the case, utilizing specific criteria or established standards. The criteria or standards may be determined by the learner or could be provided by the originator of the case study. 


\section{APPLYING THE MODEL TO CASE ANALYSIS}

A case study, as often written in a text book, is a historic account of a decision situation told through the eyes of the writer. More often than not, the writers of the cases are second hand observers or researchers of the reported scenario. While text book cases may not be ideal, there is good reason for their utilization. It is generally not feasible to have students assigned to real life companies where they could learn by both trial and error or observing others make decisions. Additionally documented, real-life decision situations are more efficient venues for teaching business decision making than book learning from typical academic prose. Harrison and St. John (2008) assert that "case analysis, to some extent, mirrors the processes managers use to make real strategic decisions. The main advantages managers have over students who analyze cases are that they have more information and more experience." Nevertheless, both must learn to make decisions without full information. (Harrion and St. John, 2008. P. 179)

Some of us who utilize the case method of learning/teaching in our classroom often experience the frustration of having student groups turn in lengthy reports or make case presentations that are replete with recitation of case facts but are void of analysis beyond what is obvious. Another major weakness often observed is the attempt on behalf of the students to mix description and prescription together without much of an attempt to diagnose, examine or evaluate the elements of the case or the relationships between the various elements. Thus the purpose of this paper is to provide a framework for teaching with the case method a marketing strategy course.

\section{Analysis Of Case Elements}

Case reports are often composed of a large number of elements. Some of the elements are explicitly presented as in history and background information about the case, the names and roles of the major role players, the decision situation facing the decision maker, past and current strategies, performance data-financial, sales, etc., information on competition, industry, customers, and other such explicitly presented communication. These rather conspicuous components are easily recognized and classified by the students as to their true nature: information about customers, competitors, the market, company performance, past strategies, and so forth. Often in his or her case analysis, the student can note with ease the type of corporate culture the company manifests, as well as develop a fair picture of the decision situation by rehashing such case information as who the customers are, where they are, and what they seem to want or not want.

There are many elements in the case report that are not so clearly labeled or identified by the case writer. Many of these elements may be of great significance to the process of identifying the case's strategic issues along with the best solutions to those issues. Unless the student is able to detect, comprehend, examine and evaluate these elements, he or she may never be able to articulate a plan or strategy that could actually achieve the managerial objectives implicated in the case.

In a typical case study, the analysis of the elements involves having the student achieve the following cognitive and behavioral objectives:

1. The ability to develop a short Historical Perspective of the case, the company and the decision situation

a. Short history of the company

b. Corporate culture: Mission, super-ordinate goal, communication networks, rites and rituals, distinctive competencies, and organizational dynamics.

c. Corporate business model

2. The ability to perform an external situation assessment:

a. Customer Analysis: Segments profile, motivation, unmet needs.

b. Competitor Analysis: Profiles, strategic groups, performance, strengths, weaknesses, and distinctive competencies.

c. Market Analysis: size, projected growth, profitability, entry barriers, cost structure, trends, Past and Current, Key Success Factors, and driving forces in the industry.

d. Environmental Analysis: Legal, Economic, Technological, Socio-Cultural, and Demographic. 
3. The ability to perform an internal Situation Assessment:

a. Performance Analysis: Profitability, sales, customer satisfaction, marketing mix assessment, employee capabilities, and skills, etc.

b. Determinants of strategic options: Past and current strategies, resource analysis, strengths and weaknesses.

4. SWOT Summary of opportunities, threats, strengths and weaknesses.

It is fair to surmise that a useful textbook case may have a number of unstated or implicit assumptions, elements, or relationships which can only be inferred from an analysis of both explicit and implicit case information to achieve the desired results from the exercise. Some of these elements are statements of facts, others may be statements of values and others may be statements of personal values or organizational norms and intent. As in the case of a medical exam, more complex analysis may be necessary to identify all the elements in the case and to assess the relationship between the various elements.

Completing this level of analysis involves an understanding of managerial decision making as well as the comprehension of the data and other information presented in the case. For the most part, the outcome of this level of analysis is for the most part descriptive with some minor deductive reasoning relative to the decisions to be made in the case.

\section{ANALYSIS OF RELATIONSHIPS}

Having identified the elements in the case, the student or decision maker still has the task of determining the relationships among the various elements as well as the relationships among the various parts of the case. The basic tasks of analysis at this level are that of identifying the strategic issues facing the organization, and the strategic options available for dealing with the strategic issues. At this level of analysis, the main question for the students to answer is: "so what?" The question should be asked to each key fact or element in the case, as well as combinations of elements that could be significant. For example, a student could ask: So what if the consumer tastes have been shifting and the company does not have any new products in the pipeline? So what if competitors have excess capacity and the company decides to increase its prices? So what if market is demanding energy-efficient products and the company does not have any in its product line?

Answering the "so what?" question demands more than the comprehension of the information in the case, and more than the mere description of the various elements identified in the case. Much of the analysis of relationships may deal with the consistency among the elements, and the relevance of the elements or key facts to the strategic issue or issues that must be addressed in the case. Strategic issues are best stated in the form of a question. For example: how can a company increase its profit if it is forced to match the prices of competition? How can a company maintain quality customer service with declining supply of qualified labor? Or how can a company meet the challenge of developing new energy efficient vehicles with nonexistent cash reserves. All of these stated issues represent relationships between two or more elements of analysis in the case. There may be a relationship between a consumer trend and an insufficient resource, between an environmental threat and a relative weakness of some sort, or between an emerging key success factor and a major strength or weakness. This level of analysis demands a lot more than mere description, and involves the use of diagnostic tests or implements.

Like most health care professionals, business strategists and decision makers must consistently perform their duties under conditions of uncertainty, and therefore they have to weigh various probabilities and possible courses of action before making crucial decisions. Many of these decisions are not straightforward because neither medicine nor business is an exact science. The factors that affect decision making have more than evidentiary value; such factors, including both social and psychological, may actually affect what decisions are put up for consideration as well as determine the ultimate form of the final decisions. 
When a physician examines a patient and hears his or her complaint, the physician will then have to make a decision on what is wrong. The diagnosis is based on answers to questions asked of the patient and on tests that are performed. Even at this stage a physician has to weigh the risks inherent in a particular diagnostic procedure against the risks of following a treatment plan without the information that would result from performing the diagnostic procedure. In prescribing treatment, the physician also knows that the treatment for one illness may aggravate another illness. Furthermore, many factors must be taken into account, such as the patient's characteristics, ability to tolerate pain, allergies to certain medications, living conditions, and etc. Physicians and health care professionals develop judgment from three sources. First, during medical training, they study anatomy and physiology to understand the various parts of the human body and the "relationships" between these parts. Second, these professionals observe during their training assessments made by people more experienced in their field. And third, health care professionals learn directly through trial and error-They make diagnosis, prescribe a treatment, and observe the results. It is important to note, however, that there is a great deal of disagreement among health care experts regarding particular treatments or the use of certain drugs for the treatment of some ailments.

As in the case of health care decisions, business professionals must understand the elements of a decision situation and the linkages between the various elements in order to make any useful diagnosis. And like medicine, diagnosing the main issues or problems to be addressed in a particular case requires the knowledge of the possible diagnostic tests, the relative costs and benefits of such tests, and the ability to interpret the results of such diagnostic tests. Because the root cause of a problem or issue could be difficult to identify, this level of analysis could require multiple tests of multiple elements and relationships. Some of these tests could be quantitative and objective. Others may involve the use of social and psychological metrics or criteria. Either way, the analysis of relationships requires going beyond the obvious. This is by far, the greatest caveat in case analysis reports or presentations by student groups. Often this section is a mere rehash of case facts and summary of case parts. Instead of repeating the fact that sales have been declining for the past five years, the students should be examining the trends to answer the question: Why have sales been declining for the past five years, and what can be done to arrest that decline or reverse it? Just as physicians distinguish symptoms from the disease itself, students should be able to identify core problems separately from their effects. Whenever data is available in the case, whether of financials, market research findings or time series data from one source or another, every effort must be made to break this data into its relevant elements and use it to help answer the question: "so what?"

\section{ANALYSIS OF ORGANIZATIONAL PRINCIPLES}

The highest level of analysis is the task of analyzing the structure and organization of the case. Rarely would a case writer state up front the underlying managerial principles or assumptions underlying the decision making situation. Thus, the reader may be unable to develop any type of prescription or treatment plan for the organization until he/she fully comprehends the purpose, point of view, antecedent, or the specific decision to be made. The purpose of business education is to help learners develop a "sense of smell" as to what the real bottom line is in a particular case.

It is imperative that the students understand why the textbook included the case in the first place. Moreover, it is essential that they identify the true "antecedent" for the decision situation. In other words, why are consultants (students) called upon to participate in this decision situation? Why now and not a month, or a year ago? What has happened inside or outside the organization to require such attention? Understanding the antecedent of the decision situation assists the consultant or student to identify the psycho-social elements that may confound the decision situation and makes him or her aware of the expectations of management with respect to the prescribed treatment plan. Here again, the best way to grow this high-level analysis skill is by trial and error in a classroom setting.

\section{SYNTHESIS AND EVALUATION}

Synthesis in learning is defined as the putting together of elements and parts so as to form a new whole. This is a process of working with elements and parts and combining them in such a way as to constitute a pattern or structure not clearly there before. (Bloom, 1956, P. 206) This form of cognitive learning provides for creative 
behavior on the part of the learner. However, it should be noted that the student does not have free reign with creative expression since generally he or she is expected to work within the limits set by specific problems, managerial principles, and methodological frameworks.

It should be noted that comprehension, application, and analysis also involve the combining of elements and the construction of meanings. However, they tend to be more partial and less complete than synthesis. Moreover, in synthesis, the student must draw upon elements from many sources and put them together into a structure or pattern not clearly there before-something new. Thus synthesis, to some extent, may well require some or all of the previous categories.

In case analysis, synthesis is best captured in the selection of strategic options, and the presentation of a specific detailed implementation plan. While no two strategists or students of strategic management would weave the same mosaic of a plan or strategy, such plans or strategies selected must be bound by information gained or deduced from the case write-up and any other relevant source of information. Thus, a useful plan or strategy must be consistent with the noted corporate culture, the SWOT summary, and the strategic issues identified. One of the most frequently observed weaknesses in student case reports is that of a prescribed plan or strategy which seems to completely ignore what has been discussed in the analysis section. Students should be questioned about where their suggested options came from, along with how will the proposed plan address the identified strategic issues and assist with the managerial decisions at hand.

In general, a synthesis is faulty if it lacks "goodness of fit" with the requirements of the decision problem. Faulty synthesis may be the result of inadequate comprehension or inadequate analysis, or both. For instance, if the decisions to be made are misidentified, or if the antecedent is improperly identified, omitting important elements of the analysis such as the impact of the environment, or the resources of the company. Focusing on irrelevant elements or applying improper tests to the elements could easily result in a faulty strategy or a faulty implementation of that strategy. If the analysis component is done properly, the synthesis of the strategy and implementation plan would be sound and relevant.

Last but not least, is Evaluation defined as "the making of judgments about the value, for some purpose, of ideas, works, solutions, methods, material, etc. It involves the use of criteria as well as standards for appraising the extent to which particulars are accurate, effective, economical, or satisfying." (Bloom, 1956, P. 207). Such judgments may be either quantitative or qualitative, and the criteria could be those determined by the students or those which are given to them.

Evaluation is placed at the top of the learning hierarchy because it requires to some extent all the other categories of behavior, however it is important to note that evaluation will in some cases be the prelude to an acquisition of new knowledge, a new attempt at comprehension or application, or a new analysis and synthesis. In the context of a case analysis, the student who attempts to analyze financial or performance based data may form a judgment about the accuracy of the data, the appropriateness of the data gathering methods or the design instrument, or even the fundamental assumptions of decisions made in the case. And although it is recognized that an individual is entitled to his/her own opinion as well as his/her own judgments about the value of specific information elements, one major purpose of business education and the use of the case method is to broaden the foundation on which judgments are made.

In the Strategic Market Management framework, students are called upon to use their judgment in evaluating the viability of strategic options under consideration, and also to develop an evaluation component that will be invoked as part of the implementation process to help assess the effectiveness or lack thereof of proposed strategies. Following are some factors that could be used in evaluating strategic options:

1. Cost/Benefit analysis: Do the financial benefits of the proposed strategy outweigh the financial costs?

2. Compatibility with the company's mission and strategic goals? Does the pursuit of a given strategy protect other company strengthen or neutralize weaknesses? 
3. Implementation viability: Will the company be able to implement such strategy in a timely manner and with ease given the demand on resources? Does the strategy fit the capabilities, organization, structure, processes, and overall culture?

4. Ethical/Moral viability: Is the strategy consistent with the core values of the organization and its major stakeholders?

5. Durability of returns: Does the viability of the strategy extend beyond the current cycle? Are there potential synergies from our selected strategy and anticipated competitive responses or emerging market or environmental forces?

In addition to the above set of metrics, the students could use such tools as Pay-off Matrix or a numeric scorekeeping system to assess the acceptability of each potential strategic alternative. The result of any evaluation utilizing these and other similar metrics should be a set of recommendations that the company should pursue.

\section{SUMMARY \& CONCLUSION}

The purpose of this paper was to explore the true nature of "analysis" when using the case method in business education. As evident in most textbooks and the general literature, Business educators have not seen it necessary to explore the true meaning of analysis and to require its true manifestation in the classroom. Accordingly, many of us go on teaching and grading at the lower levels of Bloom's Taxonomy of Learning Objectives.

This paper reminds us that proper analysis goes beyond mere comprehension, interpretation and application to involve breaking the given whole into its component elements, and assessing the relationships between the elements as well as the true purpose of the case and the general antecedent. A complete and relevant analysis should result in relevant strategic issues, and a set of relevant strategic options. Beyond analysis, the learner must be encouraged to manifest a measure of creative expression in the synthesis and integration of learned cognitions in a new whole. In the context of case analysis this involves the presentation of a set of alternative options that are internally consistent with the analysis, and a comprehensive implementation plan that incorporates a viable evaluation component.

While Bloom assigns Evaluation the top position in the hierarchy, it is nonetheless important to note that in the proper process of case analysis, comprehension, application, analysis, synthesis, and evaluation are intertwined to provide a relevant learning experience. Moreover, one should note that the process is by far more important than the report or the actual presentation, and thus we must place emphasis on assisting the students to develop higher learning skills beyond mere knowledge and comprehension. Exhibit 1 summarizes the intertwined outcomes of a strategic perspective to case analysis.

\section{REFERENCES}

1. $\quad$ Aaker, David A., Strategic Market Management, John Wiley \& Sons, Inc., 2005.

2. Bloom, Benjamin S., Editor, Taxonomy of Educational Objectives Handbook 1: Cognitive Domain., David McKay Company, Inc., New York, 1956.

3. DiMatteo, M. Robin, and Howard S. Friedman, Social Psychology and Medicine, Oelgeschlager, Gunn, \& Hain Publishers, Inc., Cambridge Massachusetts, 1982.

4. $\quad$ Harrison, Jeffrey S., and Caron H. St. John, Foundations in Strategic Management, ThomsonSouthwestern, Mason, Ohio, 2008. 


\section{EXHIBIT 1}

\section{Strategic Analysis of Marketing Cases Comprehension, Analysis, Synthesis, and Evaluation}

1. A short Historical Perspective of the case, the company and the decision situation

a. Short history of the company

b. Corporate culture: Mission, super-ordinate goal, communication networks, rites and rituals, distinctive competencies, and organizational dynamics.

c. Corporate business model

d. What is the antecedent of the decision situation?

e. What is/are the decision(s) to be made?

2. Situation Assessment---External Analysis

a. Customer Analysis: Segments profile, motivation, unmet needs.

b. Competitor Analysis: Profiles, strategic groups, performance, strengths, weaknesses, and distinctive competencies.

c. Market Analysis: size, projected growth, profitability, entry barriers, cost structure, trends, Key Success Factors.

d. Environmental Analysis: Legal, Economic, Technological, Socio-Cultural, and Demographic.

3. Situation Assessment --Internal Analysis:

a. Performance Analysis: Profitability, sales, customer satisfaction, marketing mix assessment, employee capabilities, and skills, etc.

b. Determinants of strategic options: Past and current strategies, resources, strengths and weaknesses.

4. SWOT Summary of opportunities, threats, strengths and weaknesses.

5. Identify 4-6 Strategic issues the company faces at the time of the decision situation.

6. Identify strategic alternatives to address the strategic issues identified in \#5

a. Product/Market investment strategies

b. Marketing function-specific strategies

i. $\quad$ Market segmentation strategies

ii. $\quad$ Marketing Mix strategies

iii. Assets, Competencies and Synergies.

7. Evaluate the viability of strategic alternatives to resolve the strategic issues

8. $\quad$ Select your strategy

9. Present a detailed implementation plan.
a. Be specific
b. Remember that Marketing effort costs money!
c. Remember that PEOPLE matter the most in determining the success or failure of any strategy.
d. Allow for contingencies
e. Include some form of a review or evaluation process to assess the effectiveness of strategies 